\title{
Single-shot, omni-directional x-ray scattering imaging with a laboratory source and
} single-photon localization

Dreier, Erik S.; Silvestre, Chantal; Kehres, Jan; Turecek, Daniel; Khalil, Mohamad; Hemmingsen, Jens H.; Hansen, Ole; Jakubek, Jan; Feidenhans'I, Robert Krarup; Olsen, Ulrik Lund

\section{Published in:}

Optics Letters

Link to article, DOI:

$10.1364 /$ ol.381420

Publication date:

2020

Document Version

Peer reviewed version

Link back to DTU Orbit

Citation (APA):

Dreier, E. S., Silvestre, C., Kehres, J., Turecek, D., Khalil, M., Hemmingsen, J. H., Hansen, O., Jakubek, J., Feidenhans'l, R. K., \& Olsen, U. L. (2020). Single-shot, omni-directional x-ray scattering imaging with a laboratory source and single-photon localization. Optics Letters, 45(4), 1021-1024. https://doi.org/10.1364/ol.381420

\section{General rights}

Copyright and moral rights for the publications made accessible in the public portal are retained by the authors and/or other copyright owners and it is a condition of accessing publications that users recognise and abide by the legal requirements associated with these rights.

- Users may download and print one copy of any publication from the public portal for the purpose of private study or research.

- You may not further distribute the material or use it for any profit-making activity or commercial gain

- You may freely distribute the URL identifying the publication in the public portal 


\title{
Single-shot, omni-directional X-ray scattering imaging with a laboratory source and single-photon localization
}

\author{
ERIK S. Dreier, ${ }^{1}{ }^{*}$ Chantal Silvestre, ${ }^{2}$ Jan Kehres, ${ }^{3}$ Daniel \\ TuRECeK, ${ }^{4,5}$ MOHAMAd Khalil, ${ }^{3}$ Jens H. Hemmingsen, ${ }^{2}$ Ole \\ HANSEN, ${ }^{2}$ Jan JaKubeK, ${ }^{4}$ Robert Feidenhans' ${ }^{6},{ }^{6}$ ULRIK $L$. \\ OLSEN $^{3}$ \\ ${ }^{1}$ Niels Bohr Institute, University of Copenhagen, Universitetsparken 5, 2100, Copenhagen, Denmark \\ ${ }^{2}$ DTU Nanolab, Technical University of Denmark, Ørsteds Plads, 2800, Lyngby, Denmark \\ ${ }^{3}$ Department of Physics, Technical University of Denmark, Fysikvej, 2800, Lyngby, Denmark \\ ${ }^{4}$ Advacam s.r.o., U Pergamenky 1145/12, Prague 7, Prague, 17000, Czech Republic \\ ${ }^{5}$ Center for Advanced Preclinical Imaging (CAPI), First Faculty of Medicine, Salmovska 3, Praha, 120 00, \\ Czech Republic \\ ${ }^{6}$ European XFEL GmbH, Holzkoppel 4, 22869, Schenefeld, Germany \\ *erik.dreier.chr@gmail.com
}

\begin{abstract}
:
Omni-directional, ultra small angle X-ray scattering imaging provides a method to measure the orientation of micro-structures without having to resolve them. In this letter, we use single-photon localization with the Timepix 3 chip to demonstrate the first laboratory-based implementation of single-shot, omni-directional X-ray scattering imaging with the beam-tracking technique. The setup allows for a fast and accurate retrieval of the scattering signal using a simple absorption mask. We suggest that our new approach may enable faster laboratory-based tensor tomography and could be used for energy resolved X-ray scattering imaging, useful in mitigate scattering signal artifacts caused by polychromatic illumination.
\end{abstract}

2020

The measurement of ultra small angle X-ray scattering from sub-resolution sample structures is within X-ray imaging commonly referred to as the dark-field contrast $[1,2]$. The dark-field contrast has potential within both non-destructive testing [3,4] and medical [5] applications as it enables the detection of microstructures smaller than the effective image resolution.

The dark-field contrast measures the increase in X-ray beam divergence due to a non-resolved $\mathrm{X}$-ray phase shift by sample micro-structures [6]. The signal is retrieved with multi-modal imaging techniques, which typically measures the conventional attenuation contrast simultaneously with the dark-field and the Differential Phase Contrast (DPC) perpendicular to the beam propagation direction. The dark-field is highly directional with X-ray scattering signal strongest orthogonal to the orientation of the micro-structures [7]. However, the first multi-modal imaging techniques, such as the Grating Interferrometer (GI) [1] and Edge Illumination (EI) setups [2], could only retrieve the scattering strength along one direction. For directional dark-field sensitivity, conventional EI and GI required advanced two dimensional optics and additional sample exposures $[8,9]$.

Single-shot, omni-directional dark-field imaging was first demonstrated using an advanced 2D phase grating, a high-resolution detector, and synchrotron radiation [10]. Recently, this interferometric method was generalized to laboratory sources [11]. Single-shot here refers to the measurement of DPC and dark-field signals in a single acquisition without the movement of gratings or masks. Previously, the Speckle-Tracking (ST) technique also demonstrated, single-shot, omni-directional dark-field images using a laboratory source and a random mask [12]. 


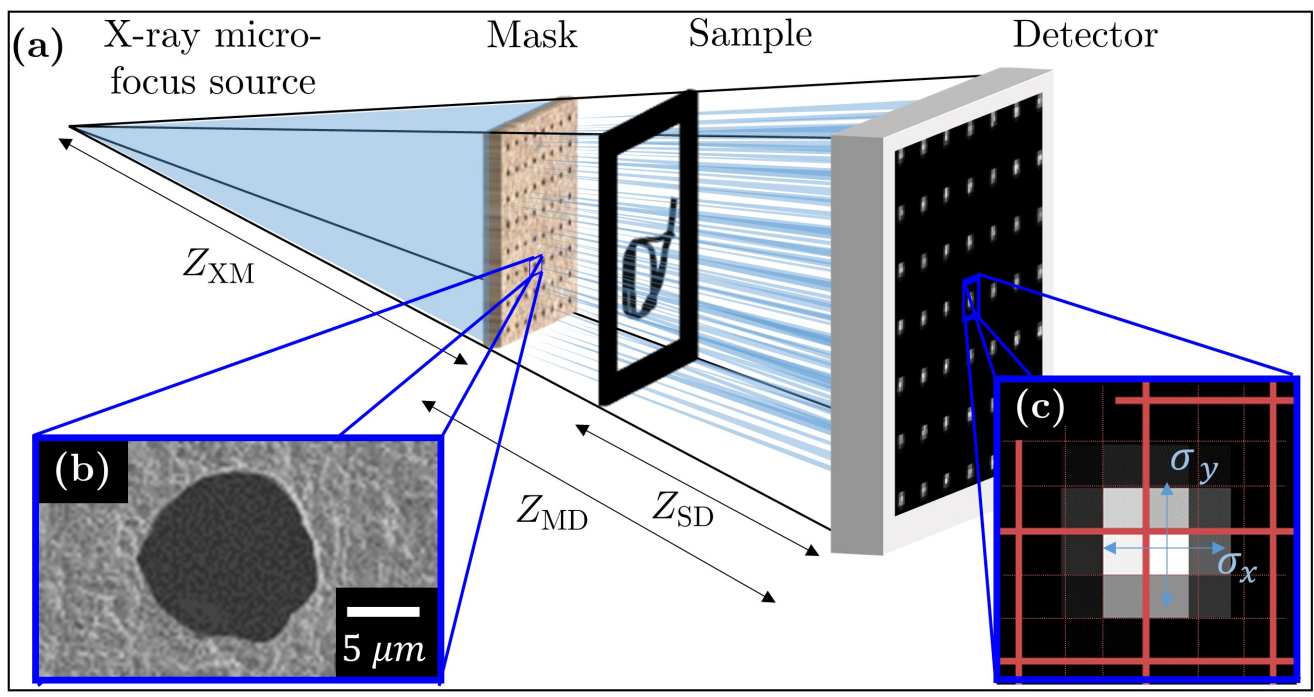

Fig. 1. The experimental setup(a): Polychromatic X-rays are emitted from a microfocus source and shaped into beamlets by the cylindrical apertures of the absorption mask [17] (b). The beamlets are attenuated, refracted, and scattered at the sample, and then detected with an Advapix detector with $1 \mathrm{~mm}$ thick silicon sensor and $55 \mu \mathrm{m}$ pixel pitch (solid red lines) using virtual subpixel (dashed red lines) (c).
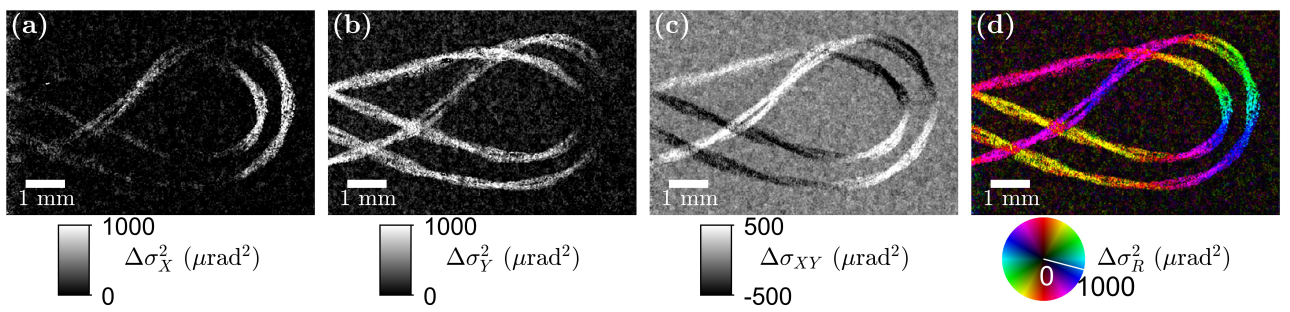

Fig. 2. Data obtained from a Kevlar fiber loop. The pixel-wise scattering function shape may be quantified by its 2 dimensional variance and covariance; $\Delta \sigma_{x}^{2}$ (a), $\Delta \sigma_{y}^{2}$ (b), and $\sigma_{x y}$ (c). From the covariance matrix, the primary scattering orientation and amplitude can be found, as illustrated in an HSV-color scheme (d).

However, the quality of the retrieved ST dark-field signal is debated and may have contributions from other effects than micro-structure scattering $[13,14]$. Furthermore, the ST technique needs long exposures to retrieve a dark-field signal due to the need for high resolution images of the speckle pattern [15].

The Beam-Tracking (BT) technique is yet another alternative for single-shot dark-field imaging. The technique uses a single, simple absorption mask to generate mutually incoherent X-ray beams. BT has been shown to render the dark-field signal more accurately than the random mask technique [14], and it imposes less restriction on the longitudinal coherence and setup geometry than the phase grating methods [16]. However, the BT technique has only been shown capable of single-shot retrieval of one dimensional dark-field signals so far.

In this letter, we demonstrate the first single-shot, omni-directional dark-field image using the BT technique. We employ the photon-counting, energy sensitive Advapix detector with the novel Timepix 3 chip [18], developed by the Medipix consortium. The chip is capable of subpixel localization of single photon events which enables the use of the Advapix detector in a compact 
BT setup [16] despite the relatively large $55 \mu \mathrm{m}$ pixel pitch. The subpixel localization of single photons has previously been applied to GI using the analog readout MÖNCH detector [19], but we are the first to apply this approach in dark-field imaging using a photon-counting detector.

Fig. 1 shows our BT setup. We use a Hamamatsu Photonics L12161-07 micro-focus source with a Tungsten target. The source was operated at $3.35 \mathrm{~W}$ with a source size of $<5 \mu \mathrm{m}$ and $U=50 \mathrm{kV}$. The measured mean energy at the detector was $14 \pm 0.5 \mathrm{keV}$. The beam is shaped into X-ray beamlets (small X-ray beams) by an absorption mask placed $Z_{\mathrm{XM}}=400 \mathrm{~mm}$ from the source. The absorption mask has a periodic array of cylindrical shaped apertures with a 100 $\mu \mathrm{m}$ pitch. It was made from a $200 \mu \mathrm{m}$ thick tungsten foil and fabricated with a combination of wet chemical etching and pico-second laser ablation, as described in details in Ref. [17]. The apertures are slightly conical with exit and entrance hole diameter of $\varnothing=9.4 \pm 0.3 \mu \mathrm{m}$ and $\emptyset=15.7 \pm 0.6 \mu \mathrm{m}$, respectively. The transmission through the $200 \mu \mathrm{m}$ tungsten is estimated to be less that $1 \%$ for X-rays with energy below $30 \mathrm{keV}$. With photons above $30 \mathrm{keV}$ constituting less than $5 \%$ of the source spectrum at the detector without a mask, the absorption mask is to good approximation fully absorbing.

With the Timepix 3 chip's fast time-stamping, each absorbed photon can accurately be localized below the pixel size at a hit rate of up to 40 Mhits s${ }^{-1} \mathrm{~cm}^{-2}$ [18] by measuring the charge sharing distribution for each event [20]. In practice, the recorded photon subpixel positions are rebinned into virtual subpixels and the beamlets' intensity distribution can afterwards be analyzed at a resolution smaller than the physical pixel size. The use of the virtual subpixels for BT is described in details in Ref. [21], where it was used for directional DPC imaging. The absorption mask is aligned such that the beamlets hit in the corners between pixels to increase the photon localization precision [21], making our setup similar to the single-mask edge-illumination technique [22]. For the presented setup, the mask to detector distance is set to $Z_{\mathrm{MD}}=480 \mathrm{~mm}$, and the mask aligned such that every fourth pixel corner of the detector is illuminated.

The sample is placed $Z_{\mathrm{SD}}=290 \mathrm{~mm}$ from the detector. By comparing the shape of the beamlets with and without sample, the presence and orientation of a scattering signal can be identified. The intensity distribution of the beamlets with a sample, can be described as a convolution of the beamlet's shape without a sample $i_{0}$ and the sample's scattering function $s$ through [16]

$$
i(x, y)=t\left(i_{0} \circledast s\right)(x-\Delta x, y-\Delta y) .
$$

$i_{0}$ is determined by the setup and can itself be described as a convolution of the mask aperture and the projected source size. $\Delta x$ and $\Delta y$ are the refraction distance perpendicular to the beamlet's propagation directions at the detector, and $t$ is the transmission through the sample. A theoretical analysis of the sample scattering function [6] relates the moments of $s$ to the differential phase signal from sub-resolution micro-structures and shows that similar information is accessible from both coherent and incoherent techniques such as GI and BT, respectively.

The beamlets' Full Width Half Maximum (FWHM) at the sample and detector are expect to be $\sim 20.5 \mu \mathrm{m}$ and $\sim 39.8 \mu \mathrm{m}$ respectively, taking into account the geometrical magnification and source blurring of the mask's entrance hole. This is within the uncertainty of the measured FWHM of beamlets at the detector of $44 \pm 3 \mu \mathrm{m}$. The size at the detector means that the beamlets can be sufficiently resolved using a rebinning of $2 \times 2$ virtual pixels per physical pixel. $2 \times 2$ virtual pixels has been shown to be the most accurate rebinning for DPC retrieval [21].

The shape of each beamlet's intensity distribution may be quantified by calculating its moments $M^{m}$, where $m$ is the order of the moment. The moments along the horizontal direction is with a 
sample given by

$$
\begin{aligned}
& M^{0}=\sum_{n}^{N} i(n) \\
& M_{x}^{1}=\frac{\sum_{n}^{N} x(n) i(n)}{M^{0}} \\
& M_{x}^{m}=\frac{\left(\sum_{n}^{N}\left(x(n)-M_{x}^{1}\right)^{m} i(n)\right)}{M^{0}} \quad \text { for } m \geq 2,
\end{aligned}
$$

and similarly for the 1 st and 2 nd moments along the vertical direction $y \cdot M^{0}=I$ is equal to the summed intensity of the beamlet with a sample. $M_{x}^{1}$ and $M_{y}^{1}$ are the beamlet's center of mass $(\langle x\rangle,\langle y\rangle)$, and the 2nd order moment is the same as the variance of the intensity distribution with $\sigma_{x}^{2}=M_{x}^{2}$ and $\sigma_{y}^{2}=M_{y}^{2}$. The covariance of the intensity distribution can likewise be calculated using

$$
\sigma_{x y}=\frac{\left(\sum_{n}^{N}\left(x(n)-M_{x}^{1}\right)\left(y(n)-M_{y}^{1}\right) i(n)\right)}{M^{0}} .
$$

A similar analysis is done for the beamlet's intensity distribution without a sample, $i_{0}$. In non directional multi-modal imaging, a common assumption is that both $i_{0}$ and $s$ are Gaussian distributions $[2,8,16]$. Similarly, we will assume that $i_{0}(x, y)$ and $s(x, y)$ can be represented by multivariate normal distributions with co-variance matrices $\boldsymbol{\Sigma}_{i_{0}}$ and $\boldsymbol{\Sigma}_{s}$. Assuming a perfectly absorbing mask, the covariance matrix of the scattering function can hence be found from

$$
\boldsymbol{\Sigma}_{S}=\boldsymbol{\Sigma}_{I}-\boldsymbol{\Sigma}_{I_{0}}=\left(\begin{array}{cc}
\Delta \sigma_{x}^{2} & \Delta \sigma_{x, y} \\
\Delta \sigma_{x, y} & \Delta \sigma_{y}^{2}
\end{array}\right),
$$

where $\Delta \sigma_{x}^{2}=\sigma_{x}^{2}-\sigma_{x, 0}^{2}$ is the difference between the beamlet's horizontal variance with and without a sample. The difference in vertical variance, $\Delta \sigma_{y}^{2}$, and covariance, $\Delta \sigma_{x, y}$ is calculated similarly. The directional refraction is likewise found from the change in the beamlet's center of mass, and the transmission from $t=I / I_{0}$. The primary scattering signal is defined as the magnitude and direction of the largest variance of $s(x, y)$. This is equal to the biggest eigenvalue of $\boldsymbol{\Sigma}_{S}$ and the direction of the corresponding eigenvector.

A kevlar fiber loop was imaged to validate the scattering retrieval method. Figure 2 shows dark-field image of the kevlar loop with a resolution of $\sim 20 \mu \mathrm{m}$, which was retrieved through raster scanning the sample in a $7 \times 7$ grid. Each sample position was measured for 30 seconds. The retrieved scattering function is rendered in an HSV color scheme with the Hue given by the orientation of $\boldsymbol{\Sigma}_{S}$ primary eigenvector, constant Saturation, and Value equal to the corresponding eigenvalue, $\Delta \sigma_{r}^{2}$. The kevlar fiber is spun from kevlar filaments of $12-15 \mu \mathrm{m}$ in diameter, which means that the individual filaments are smaller than the size of the beamlets at the sample. The filaments should cause a broadening of $s(x, y)$ perpendicular to the fiber's orientation, which is also observed in figure 2 .

Three hard wood sticks from the non coated end of wooden matches were imaged in a $6 \times 6$ grid scan for $30 \mathrm{~s}$ at each position to validate the angular precision of our technique. Hard wood is a well-known sample for multi-modal imaging as it contains structures at different length scales [16]. From a computed tomography of the wooden matches, it is identified that it has large vessels of $50 \mu \mathrm{m}$ to $100 \mu \mathrm{m}$ diameter and smaller fibers with diameter of $5 \mu \mathrm{m}$ to $25 \mu \mathrm{m}$. Both are oriented along the same direction. The sticks were placed in three different orientation as seen from radiographic attenuation image of the sticks in figure 3(a). The scattering from 

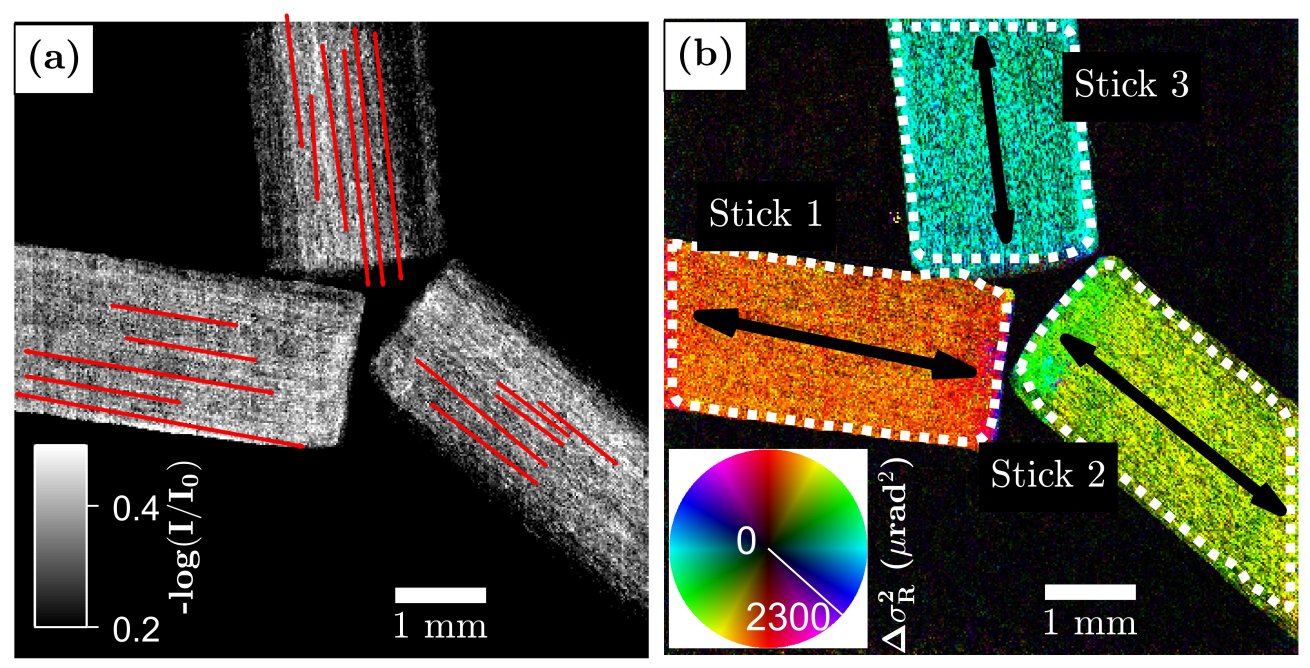

Fig. 3. The attenuation image of three hardwood sticks(a) is compared to the pixelwise orientation of the primary dark-field signal(b). The black arrows indicate direction normal to the dark-field signal, and the red lines the estimated orientation of vessels in the attenuation image.

the wooden matches is expected to be caused primarily by the fiberous structure. Hence, the orientation of the fibers can be found from the normal to the scattering signal. As illustrated in figure 3(b), the normal to the scattering function almost points along the wooden match.

To quantify the accuracy of the measured scattering angle, the orientation of the normal to the scattering signal is compared to the angle of the hard wood vessels identified in the attenuation image. The result is shown in table 1 . The predicted fiber angles from the dark-field signal scan are within 2 standard deviations of the angle found from the vessel's orientation

Table 1. The angle of hard wood vessels $\Theta_{\text {Vessel }}$, determined from the attenuation image compared to the normal to the dark-field signal from a $6 \times 6$ sample scan, $\Theta_{\text {Scat }}$.

\begin{tabular}{cccc} 
Stick & 1 & 2 & 3 \\
\hline$\Theta_{\text {Vessel }}\left({ }^{\circ}\right.$ deg. $)$ & $170 \pm 1$ & $142 \pm 2$ & $96 \pm 1$ \\
$\Theta_{\text {Scat }}\left({ }^{\circ}\right.$ deg. $)$ & $168.2 \pm 0.1$ & $142.2 \pm 0.2$ & $97.3 \pm 0.2$ \\
\hline
\end{tabular}

A butterfly was imaged to illustrate the usefulness of multi-modal imaging with directional sensitivity. The butterfly was chosen as it contains multiple different features with different size-scales. This sample was scanned in a $7 \times 7$ grid with an exposure time of $15 \mathrm{~s}$ per position. In figure 4, the butterfly's head and part of its thorax are shown using attenuation contrast, omni-directional DPC, and omni-direction dark-field contrast. The omni-directional DPC is found by the method presented in Ref. [21]. To optimize the image contrast of the thin butterfly (thickest part is $\sim 2 \mathrm{~mm}$ ), the Timepix3's spectral sensitivity was used to include only photons around the tungsten $L_{\alpha}$ emission line $\left(E_{\gamma} \in[5,12.2] \mathrm{keV}\right)$. Attenuation, DPC, and dark-field contrast all decrease with energy $[6,16]$, and hence the lower energy X-rays have larger contrast for low absorbing samples.

It is clearly seen from figure 4 how the hairy structures on the legs and the region between the butterfly's head and thorax provide a strong directional scattering signal. A similar result 
was found by Kagias et. al. [10] using their synchrotron based dark-field imaging technique. In addition, figure 4 shows how the DPC image provides an increased contrast for thin sample features with low absorption.

The Timepix 3 chip's energy sensitivity may not only be used to increase the image contrast as above, but also for actual energy resolved dark-field imaging. We anticipate that energy resolved dark-field imaging could help mitigate artifacts observed in a polychromatic X-ray dark-field signal caused by beam hardening and spectral broadening of macroscopic refraction signals [16].

By using a geometrically flexible and simple setup with no constrains on spectral coherence, the single shot, omni-directional BT method presented in this letter has similar advantages as the ST method. BT should, however, retrieve a more accurate scattering signal than ST [14]. In addition, our BT setup even seems to obtain relatively fast images when comparing the exposure time, resolution, and source specifications reported in this letter to the $\sim 180 \mu \mathrm{m}$ resolution at 1 min exposure using a $30 \mathrm{~W}$ source at $50 \mathrm{kVp}$ reported for omni-directional ST method [12]. On the other hand, it should be noted that ST directly obtains a full-field image, whereas our BT method needs a $(7 \times 7)$ grid scan of the sample to obtain full-field images, although with $\sim 20 \mu \mathrm{m}$ resolution. Faster full-field imaging could be achieved by decreasing the mask pitch to $50 \mu \mathrm{m}$ which would still provide well-spaced beamlets. The mask pitch may be reduced even further by using a multi Gaussian fitting approach to extract weak scattering signals from overlapping beamlets [23].

The setup's field of view may be increased by combining multiple Timepix 3 chips or, if energy-sensitivity is not required, using a larger, high-resolution flat-panel detector. Furthermore, the relationship between the setup's geometry and the dark-field contrast should to be studied in detail, to understand how the setup's angular sensitivity could be improved.

The BT technique has previously been demonstrated to enable tomographic reconstruction of dark-field signal using a polychromatic source [24]. Using tensor tomography, our setup may be used to reconstruct a 3D tomogram with 3 dimensional scattering tensor information in each voxel. Tensor tomography has previously been applied to traditional GI setups with 1 dimensional dark-field sensitivity, where it required 3 rotation axis [25]. We expect that our omni-directional dark-field technique makes one axis redundant, and could be used similarly to the 3D SAXS technique where tensor tomography is performed using only 2 rotation axis [26] .

In conclusion, we have presented the first single-shot, omni-directional dark-field setup using the BT method. Our results demonstrate that fast and accurate directional scattering images can be acquired using a simple, regular absorption mask. Furthermore, our results are the first demonstration of single-photon localization for dark-field imaging using a photon-counting detector. This allows for detectors with larger pixel size to be used in BT as well as energy resolved dark-field imaging, which may be useful for mitigating artifacts in the retrieved scattering signal caused by the use of polychromatic illumination.

Funding. Innovation Fund Denmark, CIL2018 project.

Disclosures. DT: 4 Corporation (E), JJ: 4 Corporation (I,E). The authors declare no conflicts of interest.

\section{References}

1. F. Pfeiffer, M. Bech, O. Bunk, P. Kraft, E. F. Eikenberry, C. Brönnimann, C. Grünzweig, and C. David, "Hard-x-ray dark-field imaging using a grating interferometer," Nat. Mater. 7, 134-137 (2008).

2. M. Endrizzi, P. C. Diemoz, T. P. Millard, J. L. Jones, R. D. Speller, I. K. Robinson, and A. Olivo, "Hard x-ray dark-field imaging with incoherent sample illumination,” Appl. Phys. Lett. 104, 024106 (2014).

3. V. Revol, I. Jerjen, C. Kottler, P. Schütz, R. Kaufmann, T. Lüthi, U. Sennhauser, U. Straumann, and C. Urban, "Sub-pixel porosity revealed by x-ray scatter dark field imaging," J. Appl. Phys. 110, 044912 (2011).

4. M. S. Nielsen, T. Lauridsen, L. B. Christensen, and R. Feidenhans'1, "X-ray dark-field imaging for detection of foreign bodies in food," Food Control. 30, 531-535 (2013). 

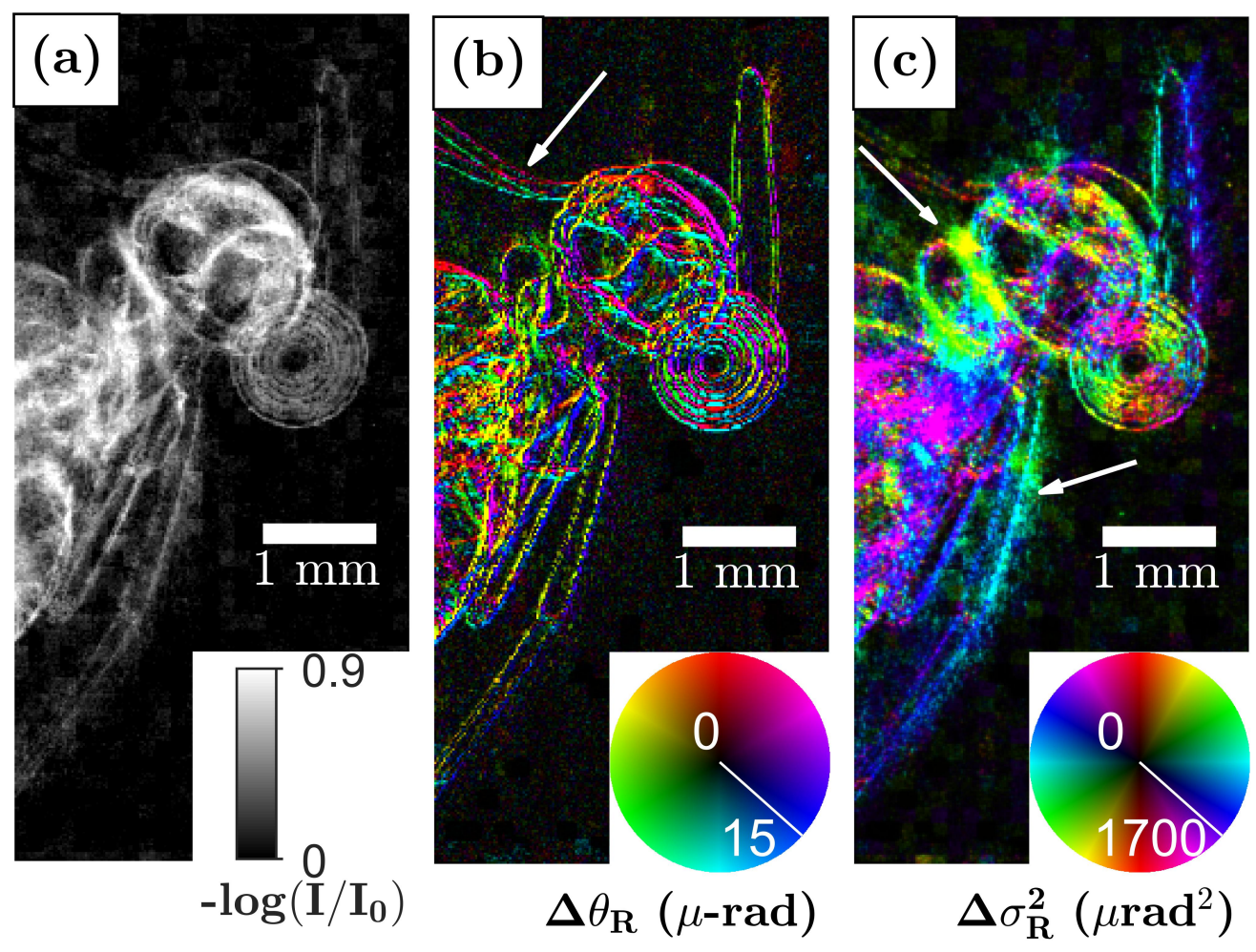

Fig. 4. Multi-modal images of a butterfly with attenuation(a), omni-directional DPC(b), and omni-directional dark-field contrast(c). Arrows indicate areas with increased contrast, such as the antennas in the DPC(b), and the hair like structure on the butterflies legs as well as the between the thorax and the head in the dark-field contrast(c).

5. P. Modregger, T. P. Cremona, C. Benarafa, J. C. Schittny, A. Olivo, and M. Endrizzi, "Small angle x-ray scattering with edge-illumination," Sci. Reports 6, 30940 (2016).

6. P. Modregger, M. Kagias, S. C. Irvine, R. Brönnimann, K. Jefimovs, M. Endrizzi, and A. Olivo, "Interpretation and utility of the moments of small-angle x-ray scattering distributions," Phys. Rev. Lett. 118, 265501 (2017).

7. T. H. Jensen, M. Bech, O. Bunk, T. Donath, C. David, R. Feidenhans'l, and F. Pfeiffer, "Directional x-ray dark-field imaging," Phys. Medicine Biol. 55, 3317-3323 (2010).

8. G. K. Kallon, M. Wesolowski, F. A. Vittoria, M. Endrizzi, D. Basta, T. P. Millard, P. C. Diemoz, and A. Olivo, "A laboratory based edge-illumination x-ray phase-contrast imaging setup with two-directional sensitivity," Appl. Phys. Lett. 107, 204105 (2015).

9. I. Zanette, T. Weitkamp, T. Donath, S. Rutishauser, and C. David, "Two-dimensional x-ray grating interferometer," Phys. Rev. Lett. 105 (2010).

10. M. Kagias, Z. Wang, P. Villanueva-Perez, K. Jefimovs, and M. Stampanoni, "2d-omnidirectional hard-X-ray scattering sensitivity in a single shot," Phys. Rev. Lett. 116, 093902 (2016)

11. M. Kagias, Z. Wang, M. E. Birkbak, E. Lauridsen, M. Abis, G. Lovric, K. Jefimovs, and M. Stampanoni, "Diffractive small angle x-ray scattering imaging for anisotropic structures," Nat. Commun. 10 (2019).

12. T. Zhou, H. Wang, and K. Sawhney, "Single-shot x-ray dark-field imaging with omnidirectional sensitivity using random-pattern wavefront modulator," Appl. Phys. Lett. 113, 091102 (2018).

13. M.-C. Zdora, "State of the art of X-ray speckle-based phase-contrast and dark-field imaging," J. Imaging 4, 60 (2018).

14. F. A. Vittoria, M. Endrizzi, and A. Olivo, "Retrieving the ultrasmall-angle x-ray scattering signal with polychromatic radiation in speckle-tracking and beam-tracking phase-contrast imaging," Phys. Rev. Appl. 7, 034024 (2017).

15. H. Wang, Y. Kashyap, and K. Sawhney, "From synchrotron radiation to lab source: advanced speckle-based x-ray imaging using abrasive paper," Sci. Reports 6, 20476 (2016).

16. F. A. Vittoria, G. K. N. Kallon, D. Basta, P. C. Diemoz, I. K. Robinson, A. Olivo, and M. Endrizzi, "Beam tracking approach for single-shot retrieval of absorption, refraction, and dark-field signals with laboratory $\mathrm{x}$-ray sources," Appl. Phys. Lett. 106, 224102 (2015). 
17. C. M. Silvestre, J. H. Hemmingsen, E. S. Dreier, J. Kehres, and O. Hansen, "Laser ablation of high-aspect-ratio hole arrays in tungsten for X-ray applications," Microelectron. Eng. 209, 60-65 (2019).

18. T. Poikela, J. Plosila, T. Westerlund, M. Campbell, M. D. Gaspari, X. Llopart, V. Gromov, R. Kluit, M. van Beuzekom, F. Zappon, V. Zivkovic, C. Brezina, K. Desch, Y. Fu, and A. Kruth, "Timepix3: a 65k channel hybrid pixel readout chip with simultaneous ToA/ToT and sparse readout," J. Instrumentation 9, C05013-C05013 (2014).

19. S. Cartier, M. Kagias, A. Bergamaschi, Z. Wang, R. Dinapoli, A. Mozzanica, M. Ramilli, B. Schmitt, M. Brückner, E. Fröjdh, D. Greiffenberg, D. Mayilyan, D. Mezza, S. Redford, C. Ruder, L. Schädler, X. Shi, D. Thattil, G. Tinti, J. Zhang, and M. Stampanoni, "Micrometer-resolution imaging using MÖNCH: towards g2-less grating interferometry," J. Synchrotron Radiat. 23, 1462-1473 (2016).

20. M. Khalil, E. S. Dreier, J. Kehres, J. Jakubek, and U. L. Olsen, "Subpixel resolution in CdTe timepix3 pixel detectors," J. Synchrotron Radiat. 25, 1650-1657 (2018).

21. E. Dreier, C. Silvestre, J. Kehres, D. Turecek, M. Khalil, J. Hemmingsen, O. Hansen, J. Jakubek, R. Feidenhans'1, and U. Olsen, "Virtual subpixel approach for single-mask phase-contrast imaging using timepix3," J. Instrumentation 14, C01011-C01011 (2019).

22. F. Krejci, J. Jakubek, and M. Kroupa, "Single grating method for low dose 1-d and 2-d phase contrast x-ray imaging," J. Instrumentation 6, C01073-C01073 (2011).

23. C. J. M. Jones, F. A. Vittoria, A. Olivo, M. Endrizzi, and P. R. T. Munro, "Retrieval of weak x-ray scattering using edge illumination," Opt. Lett. 43, 3874 (2018).

24. F. A. Vittoria, M. Endrizzi, P. C. Diemoz, A. Zamir, U. H. Wagner, C. Rau, I. K. Robinson, and A. Olivo, "X-ray absorption, phase and dark-field tomography through a beam tracking approach," Sci. Reports 5 (2015).

25. A. Malecki, G. Potdevin, T. Biernath, E. Eggl, K. Willer, T. Lasser, J. Maisenbacher, J. Gibmeier, A. Wanner, and F. Pfeiffer, "X-ray tensor tomography," EPL (Europhysics Lett. 105, 38002 (2014).

26. M. Georgiadis, M. Guizar-Sicairos, A. Zwahlen, A. J. Trüssel, O. Bunk, R. Müller, and P. Schneider, "3d scanning SAXS: A novel method for the assessment of bone ultrastructure orientation," Bone 71, 42-52 (2015). 